\title{
Obesity-induced inflammation: The impact of the hematopoietic stem cell niche
}

\author{
Emily Bowers and Kanakadurga Singer \\ Department of Pediatrics and Department of Molecular and Integrative Physiology, University of Michigan Medical School, \\ Ann Arbor, Michigan, USA.
}

\begin{abstract}
Obesity and obesity-related diseases like type 2 diabetes (T2D) are prominent global health issues; therefore, there is a need to better understand the mechanisms underlying these conditions. The onset of obesity is characterized by accumulation of proinflammatory cells, including Ly6 $\mathrm{c}^{\mathrm{hi}}$ monocytes (which differentiate into proinflammatory macrophages) and neutrophils, in metabolic tissues. This shift toward chronic, low-grade inflammation is an obese-state hallmark and highly linked to metabolic disorders and other obesity comorbidities. The mechanisms that induce and maintain increased inflammatory myelopoiesis are of great interest, with a recent focus on how obesity affects more primitive hematopoietic cells. The hematopoietic system is constantly replenished by proper regulation of hematopoietic stem and progenitor (HSPC) pools in the BM. While early research suggests that chronic obesity promotes expansion of myeloid-skewed HSPCs, the involvement of the hematopoietic stem cell (HSC) niche in regulating obesity-induced myelopoiesis remains undefined. In this review, we explore the role of the multicellular HSC niche in hematopoiesis and inflammation, and the potential contribution of this niche to the hematopoietic response to obesity. This review further aims to summarize the potential HSC niche involvement as a target of obesity-induced inflammation and a driver of obesity-induced myelopoiesis.
\end{abstract}

Conflict of interest: The authors have declared that no conflict of interest exists.

Copyright: (c) 2021, Bowers et al. This is an open access article published under the terms of the Creative Commons Attribution 4.0 International License.

Reference information: /CI Insight. 2021;6(3):e145295.

https://doi.org/10.1172/jici. insight.145295.

\section{Introduction}

The global prevalence of obesity has risen dramatically over the past decades, leading to an increased incidence of obesity-related diseases - including type 2 diabetes (T2D), cardiovascular disease, liver disease, renal failure, and cancer - and increased mortality (1-5). The prevalence of obesity has generated a strong need to better understand mechanisms that lead to obesity-induced disease. While chronic, low-grade inflammation (called metainflammation) is a known contributor to obesity-related diseases (1-7), the switch toward proinflammatory myeloid cell production is not well understood and could provide insights into therapeutic targets.

Adipose tissue (AT) expands in response to excess lipids, primarily for nutrient storage, and has a robust immune component. During steady-state, AT maintains metabolic homeostasis through communication between adipocytes and tissue-resident immune cells, the most predominant being AT macrophages (ATMs) (8). The inflammatory state of ATMs fluctuates depending on nutritional status (9-13). Acute AT inflammation following a high-fat meal is believed to contribute to AT remodeling for storage of additional lipids (14); however, during obesity, adipocytes expand, and signals drive a sustained inflammation in the AT, leading to generation of persistent inflammatory stimuli that originate from excessive adipocyte hypertrophy and further recruit monocytes to AT, thus exacerbating inflammation. This sustained inflammatory loop ultimately promotes cellular and systemic insulin resistance, as inflammatory cells and signals permeate into other tissues, including liver, pancreas, muscle, and brain (1-2, 15-17).

Expansion and accumulation of ATMs is partially due to proliferation (18-19), but it is also due to increases in circulating proinflammatory monocytes $(7,20,21)$, a finding noted in both obese patients $\left(\mathrm{CD} 14^{+}\right.$monocytes) and mice (Ly6 $\mathrm{c}^{\mathrm{hi}}$ monocytes). Circulating monocytes are produced in the BM and then migrate into tissues, via the MCP-1/CCR2 chemokine axis, where they further differentiate into inflammatory macrophages $(1,22)$. Circulating activated neutrophil populations also increase and migrate into and out of expanding AT, where they promote tissue inflammation and metabolic dysfunction (23-26). While the role of leukocytes in the promotion and propagation of inflammation in obesity-related dysfunctions continues to be covered, the mechanism driving myeloid progenitor expansion is not well understood. Here, we will discuss what is known about the factors propagating inflammation intrinsic to hematopoietic 
cells but also within the hematopoietic BM niche (Figure 1). Several gaps currently exist, providing an opportunity to further elucidate the mechanism of metainflammation.

\section{Obesity and hematopoietic stem cells}

Hematopoietic stem and progenitor cell pool fluctuations during obesity. Hematopoiesis is a tightly regulated process that allows for the continued production of the entire hematopoietic system. This process is highly dynamic and responds accordingly when exposed to different environmental stressors before returning to homeostatic hematopoiesis. As the BM is the primary site of active hematopoiesis and home to hematopoietic stem and progenitor cells (HSPCs), BM populations during obesity and chronic inflammation have been a focus of investigation (7, 27-30). Similar to AT and peripheral blood, BM exhibits induced expansion of monocytes and neutrophils in response to obesity. Interestingly, this quantitative expansion also extends to more primitive hematopoietic cells (7, 27-30). Multiple groups have shown obesity-induced increases of multiple early myeloid progenitor populations, including multipotent progenitors (MPPs), common myeloid progenitors (CMPs), pregranulocyte and macrophage progenitors (Pre-GMs), and granulocyte and macrophage progenitors (GMPs) (7, 27-30). Moreover, an increase in circulating CD34 ${ }^{+}$hematopoietic progenitors has been reported in obese patients (31).

Hematopoietic stem cells (HSCs) in obesity remain controversial, as labs have identified expansion (7, 27), reduction $(28,30)$, and no overall change (29) in HSC pool size in obese mouse models. BM transplant (BMT) studies conducted to functionally test the repopulating ability of the HSC pool in obesity have also produced conflicting data. Some groups have observed an enhanced production of inflammatory myeloid cells from high-fat diet-derived (HFD-derived) BM in both competitive and serial transplantation assays (7, 27), while others have identified impairments in HSC repopulation potential linked to altered quiescence, self-renewal, and impaired differentiation (28-30). However, a further look at these studies indicates that variability in experimental design contributes to these differences. These variations include use of genetic models of obesity (e.g., $o b / o b$ and $d b / d b$ mice) versus diet-induced obesity, models of which may also differ in the percent of calories from fat, length of diet exposure, and BMT design. It should also be noted that leptin, whose signaling is modified in $o b / o b$ and $d b / d b$ strains and elevated in obesity, has been researched as a possible regulator of the hematopoietic system. Studies going back to the 1990s have implicated leptin signaling as a positive regulator of HSC proliferation and differentiation (32-34), thus raising the question of the developmental impacts on hematopoiesis prior to the onset of obesity in an environment devoid of functional leptin or leptin receptors, as in the $o b / o b$ and $d b / d b$ mice, respectively.

Obesity, regardless of model, is marked by increased levels of a wide array of proinflammatory cytokines, like IL-6, TNF- $\alpha$, and IL-1 $\beta$, in the expanding tissues and circulation. These proinflammatory signals further promote inflammation through signaling pathways like TLR4 (35-38). While there is consensus that inflammation is a major contributor of the obesity phenotype, the exact mechanisms driving increased myelopoiesis in obese models is unclear, with both dietary components and inflammatory pathways implicated. The impact of inflammation itself on the hematopoietic system has been studied for decades, but the role of metainflammation on HSPC populations has only recently been explored (39-42). HSCs remain predominantly quiescent during steady state, with more committed downstream progenitors taking on the majority of the hematopoietic production (43). However, in response to inflammatory stressors, the HSC compartment becomes active, leading to increased proliferation and altered production of mature blood cells (44). Inflammatory signals, like IL-1 $\beta$, TNF- $\alpha$, IL-6, and TLR ligands, have all been shown to impact the HSC pool during acute and chronic inflammation and, interestingly, are also all elevated during obesity.

Inflammatory cytokines and hematopoiesis. Early research using in vitro cultures of primitive HSPCs, including human $\mathrm{CD} 34^{+}$cells and murine Lineage-Sca- $1^{+} \mathrm{CKit}^{+}$(LSK) cells, suggested a role for proinflammatory cytokines, like IL-1 $\beta$, TNF- $\alpha$, and TLR ligands, in the induction of hematopoiesis (45-47). In vitro studies have implicated IL-1 $\beta$ (48), TNF- $\alpha$ (49-52), and TLR (53-57) signaling in accelerated proliferation and myeloid differentiation of human and murine HSPCs. This enhanced HSPC proliferation and differentiation has been linked to increased expression of cell cycle activators (cyclin C, cyclin E1, cyclin-dependent kinase 4, and $M y c$ ) and myeloid lineage genes (GM-CSF receptor, PU.1, GATA-1, and C/EBP $\alpha$ ), along with reduced expression of proliferation inhibitors like $\mathrm{p} 57(48,55)$. TNF- $\alpha$ signaling has also been linked to increased HSC repopulation potential following BMT (52). While elevated IL-6 levels promote myelopoiesis and inhibit lymphopoiesis, its role during an inflammatory state is not well studied (58, 59). However, in vitro cultures and BMT studies from other groups have suggested that TNF- $\alpha$ inhibits HSPC function due to increased apoptosis and impaired self-renewal $(49,51,60,61)$. Recent work has suggested that TNF- $\alpha$ 

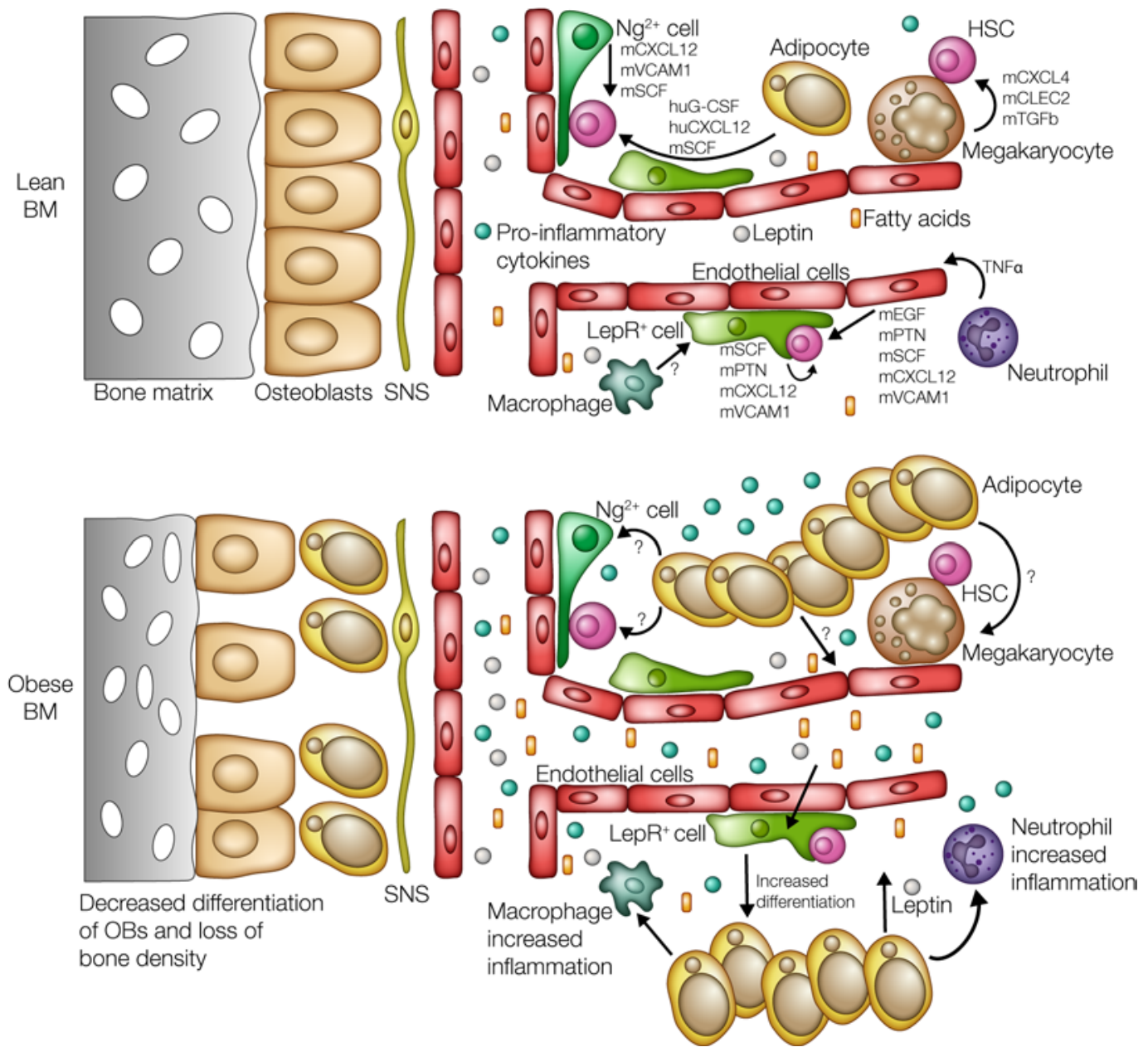

Figure 1. Obesity-induced alterations of the BM and HSC niche influence hematopoiesis. Hematopoiesis is tightly regulated by cytokines produced through the HSC niche, and research has begun to identify the individual contribution of each of these populations in the regulation and maintenance of the HSC pool (top panel). During obesity (bottom panel), the BM landscape changes dramatically. Increased production of leptin from expanding adipose tissue leads to biased differentiation of $\mathrm{LepR}^{+}$perivascular cells toward adipogenesis at the expense of osteogenesis, leading to a significant loss of bone density. While the impact of this physical expansion of the BMAT on the rest of the HSC niche and hematopoiesis is not yet known, this increase would bring many more hematopoietic and stromal cells into direct and/or closer contact with BMAT. Additionally, the expanding AT leads to increased production of proinflammatory monocytes, macrophages, and neutrophils, which in turn leads to an increase in the basal levels of inflammatory cytokines. Chronic exposure to HFD also leads to increased levels of circulating free fatty acids, which could act on both stromal and hematopoietic cells to drive and sustain production of inflammatory myelopoiesis. Illustrated by Rachel Davidowitz.

effects may be concentration dependent, as increasing TNF- $\alpha$ levels proportionally increases negative effects on HSCs (62). This dosage effect varies between different HSPC populations, as GMPs respond differently than HSCs. These data suggest that HSPC populations are differentially regulated by not just the presence or absence of TNF- $\alpha$, but also higher or lower TNF- $\alpha$ (and possibly other cytokines), within the environment.

Mechanisms of direct obesity-induced hematopoiesis. While many gaps remain in the study of inflammation in hematopoiesis, current literature suggests that activation of inflammatory pathways in HSPCs significantly influences their function, specifically in decisions concerning proliferation versus quiescence and differentiation versus self-renewal. There is also a large overlap in hematopoietic phenotypes, including enhanced myelopoiesis and HSPC expansion, observed in obesity and inflammation. Genetic loss of TLR4 signaling (via deletion of Tlr4 or downstream regulators Myd88 or Ticam1) in obese mice through whole-body KOs $(36,37)$ and BM chimeras $(35,38)$ leads to decreased levels of TNF- $\alpha$ and IL- $6(35)$, improved insulin sensitivity $(35,38)$, decreased activation of proinflammatory $\operatorname{ATMs}(36,38)$, and decreased AT fibrosis (37). Loss

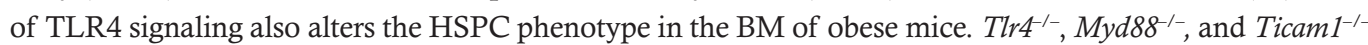
mice placed on HFD for 16 weeks had decreases in GMPs and decreased production of myeloid colonies 
via CFU assay (38). Hematopoietic-specific deletion of Tlr4 has been achieved with chimeric transplant systems generating recipient mice that contain $\mathrm{BM}$ either entirely $(7,28,32)$ or partially $(27,28,38)$ devoid of TLR4 in hematopoietic cells. Exposure of these mice to HFD decreased HSC, CMP, and GMP populations compared with the WT HFD controls. HSCs isolated from TLR4-deficient HFD-fed mice for BMT showed normal reconstitution compared with the myeloid-biased reconstitution noted from WT HFD HSCs.

Other labs have also investigated the involvement of IL-1 $\beta$ and the NLRP3 inflammasome in the propagation of myelopoiesis in obesity $(7,39) . I l 1 r^{-1-}$ mice placed on HFD for 6 months have less expansion of HSCs, CMPs, and GMPs compared with the $I l 1 r^{+/+}$HFD controls and decreased production of inflammatory cytokines from BM-derived macrophages (7). Reciprocal transplants of WT or Nlrp $3^{-1-}$ BM into irradiated $o b / o b$ recipients also suggest a role for the inflammasome in the obesity-induced BM phenotype.

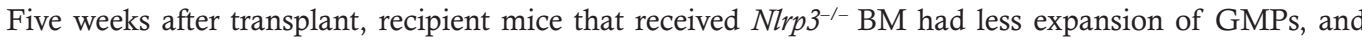
circulating monocytes and neutrophils, compared with WT BM recipients (7).

Ki67 staining has shown increased cellular proliferation of the HSC, MPP, CMP, and GMP populations of obese mice $(7,28,63)$. Obese HSPCs also exhibit consistent increases in expression of genes associated with myeloid differentiation (Csfr1, Spi1, and Runx1), myeloid activity (Stat3 and Stat6), and cell cycle activators ( $C d k 1$ and $C c n a 2)$ (28). Increased $c M y c$ expression, which promotes the cell cycle, and decreased expression of cell cycle inhibitors $p 21$ and $p 57$ have also been reported in purified obese HSCs. This gene expression profile is believed to be the result of lipid raft loss on the HSC cellular membrane, resulting in inhibited TGF- $\beta$ signaling, which has been shown to nudge HSCs out of quiescence and into proliferation (63). However, other groups have identified increased quiescence at the expense of differentiation and impaired long-term reconstitution capability in HSCs from mice exposed to chronic HFD. Increased quiescence is associated with increased Gfil and Cdkn1 expression of HSCs isolated from obese animals (29). The majority of these studies used different models, which could heavily impact the results. However, these studies shed light on the complexity of obesity and inflammation, and the dynamic changes that can occur within the hematopoietic system in response to the environment (both locally and systemically).

\section{The role of the HSC niche in hematopoiesis}

The HSC niche is a multicellular structure within the BM composed of nonhematopoietic stromal cells (adipocytes, osteoblasts [OBs], endothelial cells, perivascular mesenchymal stem cells [MSCs], and sympathetic nerves) and hematopoietic cells (megakaryocytes [Mks], macrophages, and neutrophils) that provide instructional signaling to associated HSCs. HSC niche-derived signals are important in the maintenance and proper functioning of the HSC pool during steady-state and stressed conditions, as these signals balance HSC proliferation, quiescence, differentiation, self-renewal, extravasation into circulation, and homing into the BM (64-68). While emphasis has been placed on interactions between the niche and HSCs, there is growing evidence that HSPCs residing within BM niches may also rely on these signaling pathways for proper regulation and function (65). As these populations are critical for hematopoiesis, understanding how obesity-driven inflammation impacts niche population function and the contributions of the niche to obesity-associated hematopoiesis alterations is important.

The role of BM adipocytes in hematopoiesis. Bone marrow AT (BMAT) has been an enigma in the hematopoietic and HSC niche fields. BMAT is heterogenous and can be divided into 2 populations: constitutive BMAT (cBMAT), which is densely packed and is present from birth, and regulated BMAT (rBMAT), which is more dispersed within the BM and capable of both expansion and contraction throughout life (6, 69). Studies initially linked BM adiposity with inhibited hematopoiesis based on observations that bones containing higher amounts of BMAT had less active hematopoiesis (70, 71). However, newer data point to a possible positive regulatory role for BMAT on hematopoiesis. Coculture of adipocytes and primary hematopoietic cells supports increased HSPC differentiation into mature blood cells $(72,73)$, and recent work suggests that BMAT is a source of hematopoietic and HSC regulatory cytokines. A study of human BMAT implicated this niche as a source of CXCL12 and G-CSF (74), while studies of murine BMAT suggest it is a source of SCF during hematopoietic regeneration following lethal irradiation and transplantation (75). The specific role of BMAT in the regulation of hematopoiesis is still under investigation; however, adipocyte-derived adipokines have been shown to regulate HSCs (6, 32-34, 69, 74, 76-78). Adipose-derived adiponectin promotes HSC proliferation and BMT, reconstituting potential through $\mathrm{p} 38$ MAPK activation (77) and mTORC signaling (78). Leptin, another adipokine, has also been shown to promote HSC expansion and differentiation (32-34), though the intracellular mechanism inducing proliferation is not known. 
During obesity, circulating leptin increases while adiponectin decreases. While both adipokines have been shown to promote HSC proliferation, their specific role during obesity is not known. However, expanding BMAT could provide a local source of leptin to promote HSC expansion. While scarce, some data suggest that AT-derived fatty acids could have regulatory roles in myelopoiesis $(79,80)$ and HSC pool maintenance (81). Additionally, prostaglandin $\mathrm{E}_{2}$ (PGE2), which is also elevated during obesity and derived from the fatty acid arachidonic acid, has been shown to inhibit B lymphopoiesis, suggesting another possible mechanism for increased myelopoiesis or skewing in obese BM (82).

As expanding white AT (WAT) increases production of inflammatory cytokines and receptors (8385), BMAT also increases production of inflammatory cytokines like IL- 6 , TNF- $\alpha$, and serum amyloid A3 (SAA3) (76, 86-88). In humans, BMAT expresses higher basal levels of IL-6 and TNF- $\alpha$ compared with WAT (87). Interestingly, recent data have suggested that a switch occurs during obesity wherein secretion of these factors is significantly lower in BMAT compared with expanding WAT (89). This discovery emphasizes the complexity and heterogeneity of the different AT depots, their response to obesity, and their impact on hematopoietic system regulation.

BMAT is considered a hybrid of white and brown adipocytes; however, during HFD, BMAT appears to undergo a shift toward a more WAT-like phenotype (90). Comparison of BMAT and WAT during homeostasis identified differential lipid metabolism, with decreased lipolytic activity and a shift toward cholesterol-oriented metabolism in BMAT compared with WAT (91). However, how BMAT lipid metabolism is impacted during obesity is still unknown. As WAT is typically associated with energy storage and BAT with energy expenditure, the observed metabolic shift in BMAT during HFD-induced obesity could be the result of an increased lipid storage demand. Additionally, it is not clear how this shift in metabolic signature in BMAT impacts hematopoiesis. Extensive research has gone into the identification and characterization of WAT and BAT depots during both homeostasis and metabolic dysfunction, while much less is known about the metabolic contributions of the BMAT - let alone the alterations that occur in this depot during conditions like obesity. This emphasizes the need to better understand the role of BMAT in both systemic metabolism and hematopoiesis.

The role of endothelial cells in hematopoiesis. The close proximity of HSCs to the BM vasculature was an initial clue that BM endothelial cells (BMECs) play a role in the regulation of HSCs (92-98). BMECs induce HSC proliferation and differentiation through activation of AKT (94) and the Notch signaling pathway $(95,96)$, while in vitro MAPK activation in BMECs induces significantly less expansion of hematopoietic cells (94), suggesting that various activation states within the BMEC population could have differing effects on the associated HSCs. BMECs are a major source of SCF, CXCL12 (93, 98-100), BMEC-derived EGF, and pleiotrophin (101-103), which are critical for homeostasis and hematopoietic system regeneration following BMT (97). BMECs make up a heterogenous population composed of arteriolar ECs (AECs) and sinusoidal ECs (SECs). While differences in production of all known HSC regulatory genes are not fully elucidated, AEC-derived SCF has been observed to be more critical for HSCs than SEC-derived SCF (99). HSCs also exist in different proliferative states depending on the BMEC niche. HSCs associated with AECs are found in a more quiescent state than those associated with SECs $(104,105)$; however, whether this is strictly due to the BMECs themselves or influenced by additional niche populations is not known.

BMECs express a range of TLRs (TLR1-7, TLR9) and inflammatory receptors, such as those for TNF- $\alpha$, IL-1, and IL-6 (106-108), suggesting a role in stress-induced hematopoiesis. Early in vitro assays of human EC lines identified increased expression of the myeloid differentiation cytokine GM-CSF upon IL-1 or TNF- $\alpha$ stimulation, suggesting that BMECs contribute to demand-dependent hematopoiesis $(109,110)$. In recent years, it has become apparent that G-CSF-mediated emergency granulopoiesis is heavily dependent on inflammatory signaling through BMECs, in part through TLR4 (111-114). Additionally, LPS and TNF- $\alpha$ induce expression of Jagged-2 on BMECs and Notch-1 and -2 on hematopoietic cells, suggesting that these pathways may work together to promote Notch signaling in the BM during inflammation (115). Chronic activation of the inflammatory stress-associated MAPK pathway in BMECs $(116,117)$ promotes aberrant $\mathrm{NF}-\kappa \mathrm{B}$ signaling, which is believed to drive myeloid bias in HSCs ex vivo at the expense of self-renewal (118). While the majority of this research has been within the context of acute infection and the associated immune response, several cytokine and signaling pathways (IL-1 $\beta$, TNF- $\alpha$, and TLR4) and the skewing toward myelopoiesis are also implicated in obesity, making these pathways prime targets for further analysis. While not studied during infection, expression of Del-1 by BMECs has also been shown to regulate homeostatic production of myeloid cells, suggesting a possible signaling pathway that is disrupted during obesity (119). 
Significant impairments in BMECs of the HSC niche have been observed in the context of diabetes (120-122). While some studies focus on type 1 diabetes (T1D), which addresses the impact of hyperglycemia and differs from the obese conditions associated with $\mathrm{T} 2 \mathrm{D}$, they still provide insight into the effect of metabolic dysfunction on the BM. When mice are exposed to streptozotocin to induce $\beta$ cell failure, the BM undergoes significant loss of hematopoietic cells, a dramatic decrease in vascular density, and increased vascular permeability $(120,122)$. BMECs from streptozotocin-treated mice also have increased levels of ROS and decreased migration toward chemokines CXCL12 and VEGF-A (120, 121). BMECs from these mice also exhibit decreased AKT pathway activation, which is associated with impaired BMEC migration, leukocyte extravasation, and vascular permeability (122).

The role of BMECs in metainflammation are limited, but recent work described BMEC-specific EGFR signaling in mediating the BM phenotype observed in obesity (123). The authors noted a significant reduction in CXCL12 in BMECs from obese mice; however, increased HSC localization at the BM vasculature was observed in obese mice compared with controls. These results are conflicting, as CXCL12 is considered the prominent cytokine for HSC retention in the BM, and a reduction in CXCL12 would not typically associate with increased HSC recruitment. BMEC-specific EGFR deletion in Cdh5-Cre;EGFR ${ }^{f l f l}$ mice had no effect on CXCL12 expression in response to HFD, and EGFR-deficient mice underwent increased expansion of HSCs, along with notably more myelopoiesis compared with the WT HFD group. The authors of this study conclude that BMEC EGFR signaling is protective and aids in maintenance of normal hematopoiesis. One possible caveat of this study arises from the use of Cdh5-Cre mice, as constitutive deletion in BMECs could result in deletion in the hematopoietic system (86). Overall, these data suggest that obesity and hyperglycemia influence the HSC niche by both promoting enhanced myelopoiesis and affecting mechanisms that keep hematopoiesis in check (124).

The role of perivascular stromal cells in hematopoiesis. Perivascular stromal cells are found throughout the BM space, although they are most frequently found in close association with BM vasculature $(93,104)$. Recent work has further characterized this population into, roughly, 2 distinct subtypes: leptin receptor ${ }^{+}\left(\mathrm{LepR}^{+}\right)$cells (98), which are relevant to obesity, and the $\mathrm{Ng}^{+}$population (104). Perivascular stromal cells not only show characteristics of MSCs, but also express high levels of critical HSC regulatory proteins, including SCF, CXCL12, VCAM1, and Ptn $(67,98,104,125,126)$. BM stromal cell-derived TNF- $\alpha$ has also been shown to regulate HSC biasing toward myelopoiesis via activation of angiotensin II (127). Mice lacking the angiotensin II receptor in their BM stroma have decreased production of granulocytes and macrophages and reduced GMPs (127), pointing to an involvement of the BM niche in promoting HSC skewing. Recent data have also suggested that these CXCL12 sources are important for maintenance of the MPP population (128). Thus, it is possible that the niche is not specific to HSCs but regulates hematopoiesis through multiple stages of differentiation.

BM MSCs have been implicated in regulating both the innate and adaptive immune response through the production of cytokines and inflammatory factors, such as TNF- $\alpha$, IL-10, IL-6, PGE-2, NO, and CXCL12 $(129,130)$. The effect of these cells on the immune system is quite varied, as BM MSCs can both inhibit (131-135) and promote (136-140) immune responses. MSCs can inhibit T cell responses (via IFN- $\gamma$, TNF- $\alpha$, or IL-1 $\beta$ ) (141-143) and promote antiinflammatory signaling in macrophages (via PGE-2 and TLR2) $(134,135)$. Activation of MSC TLRs promotes the increased production and secretion of proinflammatory cytokines (IL-1 $\beta$, IL-6, TNF- $\alpha$, CCL5, and type 1 IFNs; refs. 136, 137), which could increase inflammation in the BM environment. MSC-derived IL-6 promotes expansion of myeloid progenitors and mature myeloid cells $(138,139)$, and TLR4 signaling on MSCs directly leads to secretion of the monocyte chemokine MCP-1 (also known as CCL2) to promote the extravasation of inflammatory Ly6 $\mathrm{c}^{\text {hi }}$ monocytes into circulation (140), another mechanism that could be exacerbated during obesity.

We have a limited understanding of the role of perivascular MSCs during obesity-induced hematopoiesis. In T1D, BM MSCs become nonresponsive to G-CSF signaling, leading to a maintained CXCL12 expression and impaired mobilization of HSPCs (144). BM MSCs are capable of differentiating down the osteogenic or adipogenic pathways, and data taken from obese mice heavily suggest that differentiation is altered during obesity. $\mathrm{LepR}^{+}$perivascular cells are targeted by elevated leptin and differentiate into adipocytes at the expense of OBs, leading to BMAT accumulation and decreased bone density $(6,145)$. However, it is not clear if differentiation of these MSCs would lead to HSC niche loss or if this would contribute to obesity-associated hematopoietic phenotypes.

The role of the sympathetic nervous system (SNS) in hematopoiesis. The SNS innervates the BM by traveling along the arteriolar vasculature (146). The SNS helps prevent HSC exhaustion through production of 
TGF- $\beta$, which pushes HSCs into a quiescent state (146). Catecholamines produced by the SNS can directly act on HSCs or aid in the BM response to G-CSF-mediated mobilization by altering CXCL12 expression (147-151). Treatment of human CD $34^{+}$HSPCs with a dopamine agonist in the presence of G-CSF prior to BMT increased hematopoietic colonies and increased engraftment into NOD-SCID recipient mice (150).

While the use of adrenergic receptor agonists on both human and murine immune cells leads to decreased production of cytokines (TNF- $\alpha$, IL-6, IL-12, and IFN- $\gamma$ ) and increased migration $(149,152)$, there is limited information on how SNS signals affect either BM immune cells or HSPCs during inflammatory stress and obesity. Some data suggest that T1D impairs SNS-induced mobilization of HSCs following G-CSF treatment by preventing CXCL12 downregulation in BM MSCs (144). A recent model of SNS-driven atherosclerosis (a comorbidity of obesity) led to a reduction in HSC niches - like sinusoidal endothelial cells - and extravasation of HSPCs out of the BM and into the spleen, suggesting severe BM dysfunction (153). This correlated with increased production of neutrophil-derived proteases capable of targeting a critical HSPC chemokine receptor, CXCR4 (153). Treatment of these mice with an adrenergic receptor blocker prevented CXCR4 cleavage, suggesting a possible mechanism by which SNS targets neutrophils to regulate HSPC trafficking into and out of the BM. However, the impact of chronic low-grade inflammation and obesity on the SNS and its role in regulating hematopoiesis is still largely unknown and clinically important to investigate.

The role of $O B s$ in hematopoiesis. OBs are responsible for bone synthesis and mineralization, and they are capable of positively regulating hematopoiesis and HSC function $(154,155)$. In vivo murine models have suggested that OBs can modulate the size of the HSC pool through increased expression of Jagged-1 (156) and activation of BMP signaling (157). OB-derived angiogenin — another factor elevated in obesity — can promote HSC quiescence, as loss of angiogenin from osteolineage BM cells leads to increased proliferation of the HSC pool (158). However, recent studies have contradicted these data and suggest OB signaling is dispensable for proper HSC maintenance and function (159). Interestingly, CXCL12 deletion from OBs has no effect on the HSC pool but does lead to depletion of early lymphoid progenitors $(93,98)$, further supporting the existence of niches for hematopoietic progenitors. While the data might still be unclear as to the specific role of OBs in regulation of hematopoiesis, plenty of data support their role during inflammation. Following bacterial infection, OBs are a source of several inflammatory factors (e.g., IL-6, IL-8, CXCL10, and MCP-1) (160-163). Additionally, OBs express several TLRs (TLR2, TLR4, TLR5) and increase IL-6 expression when cultured in the presence of TNF- $\alpha$ or IL-1 $\beta$, suggesting an active contribution to the immune response $(161,163-165)$.

Obese patients and mice undergo significant bone loss. Studies suggest that obesity-induced bone loss is due to skewed differentiation of LepR $\mathrm{R}^{+}$cells toward adipogenesis instead of osteogenesis (145); however, several cytokines produced both by AT and immune cells can negatively impact maintenance of the OB population and bone remodeling. Leptin and adiponectin have also been implicated as regulators of bone homeostasis. Leptin has been shown to positively and negatively influence the OB pool size. Early in life, leptin is believed to stimulate bone growth; however, later in development and during obesity, it may promote bone loss through induction of RANKL expression, thereby promoting osteoclast production and bone reabsorption (166). Adiponectin is significantly reduced in obesity and appears to have a more protective role on OBs. In vitro and in vivo assays have suggested that adiponectin promotes OB production and inhibits osteoclast formation (167). Moreover, IL- 6 and TNF- $\alpha$, which are elevated during obesity, have been shown to promote osteoclast differentiation (168-170), thereby prompting bone loss. While OBs may not directly impact the HSC pool, loss of this population could negatively impact the production of lymphoid cells in obese mice and humans.

The role of Mks in hematopoiesis. Mks are large, multinucleated cells that reside in close proximity to the vasculature, where they shed platelets into circulation. As with other identified niche populations, Mks became of interest due to their close association with HSCs (171-173). Data suggest that Mks induce HSC quiescence through CXCL4, CLEC2, and TGF- $\beta$ signaling (171-173). More recently, coculture of Mk-derived microparticles with human CD34+ HSPCs appears to induce Mk lineage differentiation (174). This work on Mks was among the first to implicate a mature hematopoietic cell as a component of the HSC niche.

Mks are targeted in response to inflammation (175-177). In mice, IL-1 $\beta$ administration increases not only the size of Mks, but also circulating platelets and granulocytes $(178,179)$. IL-6 has also been shown to influence the output of platelets $(179,180)$ through increasing levels of thrombopoietin (TPO), a critical cytokine for Mk differentiation and maturation (178, 179). Mks and platelets also express TLRs (TLR1-4, TL6, and TLR9), and treatment with LPS in humans leads to an eventual increase in TPO and platelets (181-186). Platelets may produce additional inflammatory cytokines, and levels of these transcripts have 
been directly correlated to BMI. Humans with a higher BMI have elevated transcripts encoding IL-1R1, IL-6, TNF- $\alpha$, TLR2, and TLR4 (187). While platelets have been linked to inflammatory states in systemic lupus (188) and inflammatory arthritis (189), the role of platelets and Mks in the HSC niche, metainflammation, and the increased myelopoiesis seen in obesity still remains unclear.

The role of macrophages and neutrophils in hematopoiesis. Surprisingly, Mks are not the only mature hematopoietic population with HSC regulatory function. BM macrophages, and more recently neutrophils, have been implicated as indirect regulators of the HSC pool. Macrophages regulate CXCL12 expression in BM perivascular cells, which may facilitate G-CSF-induced mobilization of HSPCs into circulation (190-192). Additionally, radio-resistant $\mathrm{BM}$ macrophages locate in perivascular niches following myeloablation, where they produce PGE2 to promote HSC reconstitution and induce CXCL12 expression in BM stromal cells (193, 194). The clearance of neutrophils by BM macrophages has also been linked to altered expression of CXCL12 in the BM and extravasation of HSPCs (195). Neutrophil-derived TNF- $\alpha$ promotes regeneration of damaged BM vasculature following myeloablation, leading to increased hematopoietic reconstitution (196). While these data suggest that BM macrophages and neutrophils indirectly regulate HSCs, some data indicate that these mature immune cells directly regulate the HSC pool through histamine production (197). These studies suggest that direct mechanisms of HSC regulation by myeloid cells in hematopoiesis have yet to be identified.

BM myeloid cells are important niche components that regulate hematopoiesis. Obesity-induced inflammation is well known to induce expansion of proinflammatory monocytes and neutrophils; however, the impact of this expansion specifically in the BM has yet to be determined. Tissue-resident macrophages, and infiltrating monocytes and neutrophils, are major drivers of inflammation through production of cytokines; however, whether or not BM myeloid cells produce similar levels is not known. Therefore, these cells may promote the transition into an inflammatory BM environment through similar mechanisms. These signals could target both HSPCs and the BM niche to promote production of more inflammatory myeloid cells. While studies suggest defective acute inflammatory responses, they also raise the question as to how functional impairments of these mature myeloid cells could alter HSC regulation in the BM.

\section{Discussion}

Metainflammation is a driver for lifetime mortality and morbidity; hence, it is critical to understand the mechanisms contributing to alterations of hematopoiesis. While the majority of studies conducted have focused on the changes occurring within metabolic tissues such as the AT, the impact of obesity on hematopoiesis is still a new area of research. Several groups have begun identifying alterations to hematopoietic production during the onset of obesity in mice. More specifically, obesity promotes increased production of myeloid cells through biased expansion of myeloid-specific progenitors and skewing of the HSC pool toward myelopoiesis. However, there are gaps in understanding the mechanisms underlying these processes and the impact of obesity on hematopoiesis and the HSC pool (Figure 1).

Going forward, it will be important to further determine if there is a dependence on specific dietary factors, such as saturated fats, or if genetic obesity itself can mimic what is observed in dietary obesity. In addition, the age of mice used in studies is another confounding factor; therefore, understanding the immune changes in both young and aged animals is clinically relevant and necessary, as seen with regard to age-related influence of metainflammation observed in recent pandemic infections (198). The use of either HSPC frequency or cell number are also important to consider in study design and data analysis. Given that cellularity itself might significantly shift in the BM space with obesity and HFD exposure, the use of frequency could be misleading. For example, expansion of a population like neutrophils (one of the most abundant blood cells in the BM) could drastically alter BM cellularity, which would significantly affect the frequency of HSCs, even if the total number of HSCs was unchanged. While this review has focused on the phenotypes attributed to obesity, it is critical to mention that all of these studies utilize male mice, as female mice do not present the same inflammatory signatures (199) or metabolic impairment. A very similar phenotype is also observed in humans, suggesting a possible effect of sex hormones. The study of the HSC niche in female BM could provide critical insight into the regulatory roles of these populations in the chronic inflammatory response.

The HSC niche in obesity has yet to be heavily interrogated. While some studies suggest that obesity impairs BMEC function, increases BM adiposity, and decreases bone density, the impact of these changes on the ability of the niche to properly regulate hematopoiesis remains to be determined. Recent work demonstrates that adipocytes and ECs can communicate through extracellular vesicles (200). Such com- 
munication would have large implications into the role of BMAT as a signaling hub in the BM space that could influence hematopoietic decisions.

Given that inflammation is so heavily linked to the pathogenesis of obesity, understanding the cell types within the hematopoietic niche that support or impair hematopoiesis is critical. Focused research into the signals and the role of inflammation in the regulation of hematopoiesis through the HSC niche is essential to understand the therapeutic potential of altering the BM niche to regulate immune responses and improve metabolic health in obesity.

\section{Acknowledgments}

We would like to thank members of the Singer lab that participated in review of this manuscript. EB is supported by the AHA Postdoctoral Fellowship 20POST35120473, and KS is supported by NIH/NIDDK R01DK11583.

Address correspondence to: Kanakadurga Singer, Department of Pediatrics, Division of Pediatric Endocrinology, D1205 MPB,1500 E Medical Center Dr., Ann Arbor, Michigan 48109, USA. Phone: 734.764.5175; Email: ksinger@umich.edu.

1. Kanda H, et al. MCP-1 contributes to macrophage infiltration into adipose tissue, insulin resistance, and hepatic steatosis in obesity. J Clin Invest. 2006;116(6):1494-1505.

2. Morris DL. Minireview: emerging concepts in islet macrophage biology in type 2 diabetes. Mol Endocrinol. 2015;29(7):946-962.

3. Doerstling SS, et al. Obesity and cancer metabolism: a perspective on interacting tumor-intrinsic and extrinsic factors. Front Oncol. 2017;7:216

4. Rhee CM, et al. The dual roles of obesity in chronic kidney disease: a review of the current literature. Curr Opin Nephrol Hypertens. 2016;25(3):208-216.

5. Berg AH, Scherer PE. Adipose tissue, inflammation, and cardiovascular disease. Circ Res. 2005;96(9):939-949

6. Borouman P, Klip A. Bone marrow adipose cells — cellular interactions and changes with obesity. J Cell Sci. 2020;133(5):jcs238394

7. Nagareddy PR, et al. Adipose tissue macrophages promote myelopoiesis and monocytosis in obesity. Cell Metab. 2014;19(5):821-835.

8. Wouters K, et al. Circulating classical monocytes are associated with CD11 $\mathrm{c}^{+}$macrophages in human visceral adipose tissue. Sci Rep . 2017;7:42665.

9. Lumeng CN, et al. Obesity induces a phenotypic switch in adipose tissue macrophage polarization. J Clin Invest. 2007;117(1):175-184

10. Lumeng CN, et al. Increased inflammatory properties of adipose tissue macrophages recruited during diet-induced obesity. Diabetes. 2007;56(1):16-23.

11. Kosteli A, et al. Weight loss and lipolysis promote a dynamic immune response in murine adipose tissue. J Clin Invest. 2010;120(10):3466-3479.

12. Shaul ME, et al. Dynamic, M2-like remodeling phenotypes of CD11c+ adipose tissue macrophages during high-fat diet--induced obesity in mice. Diabetes. 2010;59(5):1171-1181.

13. Zeyda M, et al. Newly identified adipose tissue macrophage populations in obesity with distinct chemokine and chemokine receptor expression. Int J Obes (Lond). 2010;34(12):1684-1694.

14. Wernstedt Asterholm I, et al. Adipocyte inflammation is essential for healthy adipose tissue expansion and remodeling. Cell Metab. 2014;20(1):103-118.

15. Odegaard JI, et al. Alternative M2 activation of Kupffer cells by PPARdelta ameliorates obesity-induced insulin resistance. Cell Metab. 2008;7(6):496-507.

16. Wu H, Ballantyne CM. Skeletal muscle inflammation and insulin resistance in obesity. J Clin Invest. 2017;127(1):43-54.

17. Guillemot-Legris O, Muccioli GG. Obesity-induced neuroinflammation: beyond the hypothalamus. Trends Neurosci. 2017;40(4):237-253.

18. Amano SU, et al. Local proliferation of macrophages contributes to obesity-associated adipose tissue inflammation. Cell Metab. 2014;19(1):162-171.

19. Zheng C, et al. Local proliferation initiates macrophage accumulation in adipose tissue during obesity. Cell Death Dis. $2016 ; 7: 2167$.

20. Kullo IJ, Hensrud DD, Allison TG. Comparison of numbers of circulating blood monocytes in men grouped by body mass index $(<25,25$ to $<30$, $>$ or $=30)$. Am J Cardiol. 2002;89(12):1441-1443.

21. Friedrich K, et al. Perturbation of the monocyte compartment in human obesity. Front Immunol. 2019;10:1874.

22. Kamei N, et al. Overexpression of monocyte chemoattractant protein-1 in adipose tissues causes macrophage recruitment and insulin resistance. J Biol Chem. 2006;281(36):26602-26614.

23. Ohshita K, et al. Elevated white blood cell count in subjects with impaired glucose tolerance. Diabetes Care. 2004;27(2):491-496.

24. Brotfain E, et al. Neutrophil functions in morbidly obese subjects. Clin Exp Immunol. 2015;181(1):156-163.

25. Talukdar S, et al. Neutrophils mediate insulin resistance in mice fed a high-fat diet through secreted elastase. Nat Med. 2012;18(9):1407-1412.

26. Elgazar-Carmon V, et al. Neutrophils transiently infiltrate intra-abdominal fat early in the course of high-fat feeding. J Lipid Res. 2008;49(9):1894-1903.

27. Singer K, et al. Diet-induced obesity promotes myelopoiesis in hematopoietic stem cells. Mol Metab. 2014;3(6):664-675.

28. Liu A, et al. Bone marrow lympho-myeloid malfunction in obesity requires precursor cell-autonomous TLR4. Nat Commun. 2018;9(1):708.

29. Lee JM, et al. Obesity alters the long-term fitness of the hematopoietic stem cell compartment through modulation of Gfil expression. J Exp Med. 2018;215(2):627-644. 
30. van den Berg SM, et al. Diet-induced obesity in mice diminishes hematopoietic stem and progenitor cells in the bone marrow. FASEB J. 2016;30(5):1779-1788

31. Bellows CF, et al. Influence of BMI on level of circulating progenitor cells. Obesity (Silver Spring). 2011;19(8):1722-1726.

32. Dias CC, et al. A synthetic fragment of leptin increase hematopoietic stem cell population and improve its engraftment ability. $J$ Cell Biochem. 2015;116(7):1334-1340.

33. Umemoto Y, et al. Leptin stimulates the proliferation of murine myelocytic and primitive hematopoietic progenitor cells. Blood. 1997;1(90):3438-3443

34. Claycombe K, et al. A role for leptin in sustaining lymphopoiesis and myelopoiesis. Proc Natl Acad Sci U S A. 2008;105(6):2017-2021

35. Saberi M, et al. Hematopoietic cell-specific deletion of toll-like receptor 4 ameliorates hepatic and adipose tissue insulin resistance in high-fat-fed mice. Cell Metab. 2009;10(5):419-429.

36. Orr JS, et al. Toll-like receptor 4 deficiency promotes the alternative activation of adipose tissue macrophages. Diabetes. 2012;61(11):2718-2727.

37. Vila IK, et al. Immune cell Toll-like receptor 4 mediates the development of obesity- and endotoxemia-associated adipose tissue fibrosis. Cell Rep. 2014;7(4):1116-1129.

38. Griffin C, et al. TLR4, TRIF, and MyD88 are essential for myelopoiesis and CD11 ${ }^{+}$adipose tissue macrophage production in obese mice. J Biol Chem. 2018;293(23):8775-8786.

39. Pietras EM. Inflammation: a key regulator of hematopoietic stem cell fate in health and disease. Blood. 2017;130(15):1693-1698 40. Boettcher S, Manz MG. Regulation of inflammation- and infection-driven hematopoiesis. Trends Immunol. 2017;38(5):345-357.

41. Mirantes C, et al. Pro-inflammatory cytokines: emerging players regulating HSC function in normal and diseased hematopoiesis. Exp Cell Res. 2014;329(2):248-254.

42. Clapes T, et al. Stress and non-stress roles of inflammatory signals during HSC emergence and maintenance. Front Immunol. 2016;7:487.

43. Sun J, et al. Clonal dynamics of native haematopoiesis. Nature. 2014;514(7522):322-327.

44. Wilson A, et al. Hematopoietic stem cells reversibly switch from dormancy to self-renewal during homeostasis and repair. Cell. 2008;135(6):1118-1129.

45. Heimfeld S, et al. The in vitro response of phenotypically defined mouse stem cells and myeloerythroid progenitors to single or multiple growth factors. Proc Natl Acad Sci U S A. 1991;88(21):9902-9906.

46. Fibbe WE, et al. Proliferation of myeloid progenitor cells in human long-term bone marrow cultures is stimulated by interleukin-1 beta. Blood. 1988;72(4):1242-1247.

47. Brugger W, et al. Ex vivo expansion of enriched peripheral blood CD34+ progenitor cells by stem cell factor, interleukin-1 beta (IL-1 beta), IL-6, IL-3, interferon-gamma, and erythropoietin. Blood. 1993;81(10):2579-2584

48. Pietras EM, et al. Chronic interleukin-1 exposure drives haematopoietic stem cells towards precocious myeloid differentiation at the expense of self-renewal. Nat Cell Biol. 2016;18(6):607-618.

49. Dybedal I, et al. Tumor necrosis factor (TNF)-mediated activation of the p55 TNF receptor negatively regulates maintenance of cycling reconstituting human hematopoietic stem cells. Blood. 2001;98(6):1782-1791.

50. Caux C, et al. Tumor necrosis factor-alpha strongly potentiates interleukin-3 and granulocyte-macrophage colony-stimulating factor-induced proliferation of human CD34+ hematopoietic progenitor cells. Blood. 1990;75(12):2292-2298.

51. Pronk CJ, et al. Tumor necrosis factor restricts hematopoietic stem cell activity in mice: involvement of two distinct receptors. J Exp Med. 2011;208(8):1563-1570.

52. Pearl-Yafe M, et al. Tumor necrosis factor receptors support murine hematopoietic progenitor function in the early stages of engraftment. Stem Cells. 2010;28(7):1270-1280.

53. Nagai Y, et al. Toll-like receptors on hematopoietic progenitor cells stimulate innate immune system replenishment. Immunity. 2006;24(6):801-812.

54. Sioud M, et al. Signaling through toll-like receptor 7/8 induces the differentiation of human bone marrow CD34+ progenitor cells along the myeloid lineage. J Mol Biol. 2006;364(5):945-954

55. De Luca K, et al. The TLR1/2 agonist PAM(3)CSK(4) instructs commitment of human hematopoietic stem cells to a myeloid cell fate. Leukemia. 2009;23(11):2063-2074.

56. Liu A, et al. Cutting edge: hematopoietic stem cell expansion and common lymphoid progenitor depletion require hematopoieticderived, cell-autonomous TLR4 in a model of chronic endotoxin. J Immunol. 2015;195(6):2524-2528.

57. Takizawa $\mathrm{H}$, et al. Pathogen-induced TLR4-TRIF innate immune signaling in hematopoietic stem cells promotes proliferation but reduces competitive fitness. Cell Stem Cell. 2017;21(2):225-240.

58. Maeda K, et al. IL-6 blocks a discrete early step in lymphopoiesis. Blood. 2005;106(3):879-885.

59. Reynaud D, et al. IL-6 controls leukemic multipotent progenitor cell fate and contributes to chronic myelogenous leukemia development. Cancer Cell. 2011;20(5):661-673.

60. Selleri C, et al. Interferon-gamma and tumor necrosis factor-alpha suppress both early and late stages of hematopoiesis and induce programmed cell death. J Cell Physiol. 1995;165(3):538-546.

61. Broxmeyer HE, et al. The suppressive influences of human tumor necrosis factors on bone marrow hematopoietic progenitor cells from normal donors and patients with leukemia: synergism of tumor necrosis factor and interferon-gamma. J Immunol. 1986;136(12):4487-4495.

62. Yamashita M, Passegue E. TNF- $\alpha$ coordinates hematopoietic stem cell survival and myeloid regeneration. Cell Stem Cell. 2019;25(3):357-372

63. Hermetet F, et al. High-fat diet disturbs lipid raft/TGF-beta signaling-mediated maintenance of hematopoietic stem cells in mouse bone marrow. Nat Commun. 2019;10(1):523

64. Pinho S, Frenette PS. Haematopoietic stem cell activity and interactions with the niche. Nat Rev Mol Cell Biol. 2019;20(5):303-320

65. Wei Q, Frenette PS. Niches for hematopoietic stem cells and their progeny. Immunity. 2018;48(4):632-648.

66. Gao X, et al. The hematopoietic stem cell niche: from embryo to adult. Development. 2018;145(2):dev139691.

67. Asada N, et al. Differential cytokine contributions of perivascular haematopoietic stem cell niches. Nat Cell Biol. 2017;19(3):214-223.

68. Joseph C, et al. Deciphering hematopoietic stem cells in their niches: a critical appraisal of genetic models, lineage tracing, and imaging strategies. Cell Stem Cell. 2013;13(5):520-533. 
69. Scheller EL, et al. Marrow adipose tissue: trimming the fat. Trends Endocrinol Metab. 2016;27(6):392-403.

70. Naveiras $\mathrm{O}$, et al. Bone-marrow adipocytes as negative regulators of the haematopoietic microenvironment. Nature. 2009;460(7252):259-263.

71. Zhu RJ, et al. Hematopoietic recovery following chemotherapy is improved by BADGE-induced inhibition of adipogenesis Int J Hematol. 2013;97(1):58-72.

72. Spindler TJ, et al. Adipocytic cells augment the support of primitive hematopoietic cells in vitro but have no effect in the bone marrow niche under homeostatic conditions. Stem Cells Dev. 2014;23(4):434-441.

73. Corre J, et al. Human bone marrow adipocytes support complete myeloid and lymphoid differentiation from human CD34 cells Br J Haematol. 2004;127(3):344-347.

74. Mattiucci D, et al. Bone marrow adipocytes support hematopoietic stem cell survival. J Cell Physiol. 2018;233(2):1500-1511.

75. Zhou BO, et al. Bone marrow adipocytes promote the regeneration of stem cells and haematopoiesis by secreting SCF. Nat Cell Biol. 2017;19(8):891-903.

76. Liu LF, et al. Characterization of age-related gene expression profiling in bone marrow and epididymal adipocytes. BMC Genomics. 2011;12:212.

77. DiMascio L, et al. Identification of adiponectin as a novel hemopoietic stem cell growth factor. J Immunol. 2007;178(6):3511-3520.

78. Masamoto Y, et al. Adiponectin enhances quiescence exit of murine hematopoietic stem cells and hematopoietic recovery through mTORC1 potentiation. Stem Cells. 2017;35(7):1835-1848.

79. Nicholls HT, et al. Hematopoietic cell-restricted deletion of CD36 reduces high-fat diet-induced macrophage infiltration and improves insulin signaling in adipose tissue. Diabetes. 2011;60(4):1100-1110.

80. Chen L, et al. Identification of CD13+CD36+ cells as a common progenitor for erythroid and myeloid lineages in human bone marrow. Exp Hematol. 2007;35(7):1047-1055

81. Ito K, et al. A PML-PPAR- $\delta$ pathway for fatty acid oxidation regulates hematopoietic stem cell maintenance. Nat Med. 2012;18(9):1350-1358

82. Shimozato T, Kincade PW. Prostaglandin E2 and stem cell factor can deliver opposing signals to B lymphocyte precursors Cell Immunol. 1999;198(1):21-29.

83. Berg AH, et al. Adipocyte differentiation induces dynamic changes in NF-kappaB expression and activity. Am J Physiol Endocrinol Metab. 2004;287(6):E1178-E1188.

84. Lin Y, et al. The lipopolysaccharide-activated toll-like receptor (TLR)-4 induces synthesis of the closely related receptor TLR-2 in adipocytes. J Biol Chem. 2000;275(32):24255-24263.

85. Rajala MW, Scherer PE. Minireview: the adipocyte--at the crossroads of energy homeostasis, inflammation, and atherosclerosis Endocrinology. 2003;144(9):3765-3773.

86. Lin Y, et al. Hyperglycemia-induced production of acute phase reactants in adipose tissue. J Biol Chem. 2001;276(45):42077-42083.

87. Miggitsch C, et al. Human bone marrow adipocytes display distinct immune regulatory properties. EBioMedicine. 2019;46:387-398

88. Li Y, et al. The unique metabolic characteristics of bone marrow adipose tissue. Front Endocrinol (Lausanne). 2019;10:69.

89. Tencerova M, et al. High-fat diet-induced obesity promotes expansion of bone marrow adipose tissue and impairs skeletal stem cell functions in mice. J Bone Miner Res. 2018;33(6):1154-1165.

90. Krings A, et al. Bone marrow fat has brown adipose tissue characteristics, which are attenuated with aging and diabetes. Bone. 2012;50(2):546-552.

91. Attane C, et al. Human bone marrow is comprised of adipocytes with specific lipid metabolism. Cell Rep. 2020;30(4):949-958

92. Kiel MJ, et al. SLAM family receptors distinguish hematopoietic stem and progenitor cells and reveal endothelial niches for stem cells. Cell. 2005;121(7):1109-1121.

93. Ding L, Morrison SJ. Haematopoietic stem cells and early lymphoid progenitors occupy distinct bone marrow niches. Nature. 2013;495(7440):231-235.

94. Kobayashi $\mathrm{H}$, et al. Angiocrine factors from Akt-activated endothelial cells balance self-renewal and differentiation of haematopoietic stem cells. Nat Cell Biol. 2010;12(11):1046-1056.

95. Butler JM, et al. Endothelial cells are essential for the self-renewal and repopulation of Notch-dependent hematopoietic stem cells. Cell Stem Cell. 2010;6(3):251-264

96. Poulos MG, et al. Endothelial Jagged-1 is necessary for homeostatic and regenerative hematopoiesis. Cell Rep. 2013;4(5):1022-1034.

97. Hooper AT, et al. Engraftment and reconstitution of hematopoiesis is dependent on VEGFR2-mediated regeneration of sinusoidal endothelial cells. Cell Stem Cell. 2009;4(3):263-274.

98. Ding L, et al. Endothelial and perivascular cells maintain haematopoietic stem cells. Nature. 2012;481(7382):457-462.

99. Xu C, et al. Stem cell factor is selectively secreted by arterial endothelial cells in bone marrow. Nat Commun. 2018;9(1):2449.

100. Ramalingam P, et al. Regulation of the hematopoietic stem cell lifecycle by the endothelial niche. Curr Opin Hematol. 2017;24(4):289-299.

101. Doan PL, et al. Epidermal growth factor regulates hematopoietic regeneration after radiation injury. Nat Med. 2013;19(3):295-304. 102. Himburg HA, et al. Pleiotrophin regulates the expansion and regeneration of hematopoietic stem cells. Nat Med. 2010;16(4):475-482.

103. Himburg HA, et al. Distinct bone marrow sources of pleiotrophin control hematopoietic stem cell maintenance and regeneration. Cell Stem Cell. 2018;23(3):370-381.

104. Kunisaki Y, et al. Arteriolar niches maintain haematopoietic stem cell quiescence. Nature. 2013;502(7473):637-643.

105. Itkin T, et al. Distinct bone marrow blood vessels differentially regulate haematopoiesis. Nature. 2016;532(7599):323-328.

106. Khakpour S, et al. Vascular endothelial cell Toll-like receptor pathways in sepsis. Innate Immun. 2015;21(8):827-846.

107. Pober JS, Sessa WC. Evolving functions of endothelial cells in inflammation. Nat Rev Immunol. 2007;7(10):803-815

108. Yao L, et al. Bone marrow dysfunction in mice lacking the cytokine receptor gp 130 in endothelial cells. Blood. 2005;106(13):4093-4101.

109. Sieff CA, et al. Interleukin 1 induces cultured human endothelial cell production of granulocyte-macrophage colony-stimulating factor. J Clin Invest. 1987;79(1):48-51.

110. Broudy VC, et al. Tumor necrosis factor type alpha stimulates human endothelial cells to produce granulocyte/macrophage colony-stimulating factor. Proc Natl Acad Sci U S A. 1986;83(19):7467-7471. 
111. Boettcher S, et al. Cutting edge: LPS-induced emergency myelopoiesis depends on TLR4-expressing nonhematopoietic cells. J Immunol. 2012;188(12):5824-5828.

112.Boettcher S, et al. Endothelial cells translate pathogen signals into G-CSF-driven emergency granulopoiesis. Blood. 2014;124(9):1393-1403.

113. Andonegui G, et al. Mice that exclusively express TLR4 on endothelial cells can efficiently clear a lethal systemic Gram-negative bacterial infection. J Clin Invest. 2009;119(7):1921-1930.

114. Andonegui G, et al. Endothelium-derived Toll-like receptor-4 is the key molecule in LPS-induced neutrophil sequestration into lungs. J Clin Invest. 2003;111(7):1011-1020.

115. Fernandez L, et al. Tumor necrosis factor-alpha and endothelial cells modulate Notch signaling in the bone marrow microenvironment during inflammation. Exp Hematol. 2008;36(5):545-558.

116. Roth Flach RJ, et al. Endothelial protein kinase MAP4K4 promotes vascular inflammation and atherosclerosis. Nat Commun. 2015;6:8995.

117. Sanchez A, et al. Map3k8 controls granulocyte colony-stimulating factor production and neutrophil precursor proliferation in lipopolysaccharide-induced emergency granulopoiesis. Sci Rep. 2017;7(1):5010.

118. Ramalingam P, et al. Chronic activation of endothelial MAPK disrupts hematopoiesis via NFKB dependent inflammatory stress reversible by SCGF. Nat Commun. 2020;11(1):666.

119. Mitroulis I, et al. Secreted protein Del-1 regulates myelopoiesis in the hematopoietic stem cell niche. J Clin Invest. 2017;127(10):3624-3639.

120. Oikawa A, et al. Diabetes mellitus induces bone marrow microangiopathy. Arterioscler Thromb Vasc Biol. 2010;30(3):498-508.

121. Hazra S, et al. Long-term type 1 diabetes influences haematopoietic stem cells by reducing vascular repair potential and increasing inflammatory monocyte generation in a murine model. Diabetologia. 2013;56(3):644-653.

122. Mangialardi G, et al. Diabetes causes bone marrow endothelial barrier dysfunction by activation of the RhoA-Rho-associated kinase signaling pathway. Arterioscler Thromb Vasc Biol. 2013;33(3):555-564.

123. Hoyer FF, et al. Bone marrow endothelial cells regulate myelopoiesis in diabetes mellitus. Circulation. 2020;142(3):244-258.

124. Flynn MC, et al. Transient intermittent hyperglycemia accelerates atherosclerosis by promoting myelopoiesis. Circ Res. 2020;127(7):877-892.

125. Mendez-Ferrer S, et al. Mesenchymal and haematopoietic stem cells form a unique bone marrow niche. Nature. 2010;466(7308):829-834.

126. Pinho S, et al. PDGFR $\alpha$ and CD51 mark human nestin+ sphere-forming mesenchymal stem cells capable of hematopoietic progenitor cell expansion. J Exp Med. 2013;210(7):1351-1367.

127. Tsubakimoto $Y$, et al. Bone marrow angiotensin AT1 receptor regulates differentiation of monocyte lineage progenitors from hematopoietic stem cells. Arterioscler Thromb Vasc Biol. 2009;29(10):1529-1536.

128. Cordeiro Gomes A, et al. Hematopoietic stem cell niches produce lineage-instructive signals to control multipotent progenitor differentiation. Immunity. 2016;45(6):1219-1231.

129. Keating A. Mesenchymal stromal cells: new directions. Cell Stem Cell. 2012;10(6):709-716.

130. Bunnell BA. New concepts on the immune modulation mediated by mesenchymal stem cells. Stem Cell Res Ther. 2010;1(5):34.

131. Ren G, et al. Mesenchymal stem cell-mediated immunosuppression occurs via concerted action of chemokines and nitric oxide. Cell Stem Cell. 2008;2(2):141-150.

132. Opitz CA, et al. Toll-like receptor engagement enhances the immunosuppressive properties of human bone marrow-derived mesenchymal stem cells by inducing indoleamine-2,3-dioxygenase-1 via interferon-beta and protein kinase R. Stem Cells. 2009;27(4):909-919.

133. Ren G, et al. Species variation in the mechanisms of mesenchymal stem cell-mediated immunosuppression. Stem Cells. 2009;27(8):1954-1962.

134. Choi H, et al. Anti-inflammatory protein TSG-6 secreted by activated MSCs attenuates zymosan-induced mouse peritonitis by decreasing TLR2/NF-kB signaling in resident macrophages. Blood. 2011;118(2):330-338.

135. Nemeth $\mathrm{K}$, et al. Bone marrow stromal cells attenuate sepsis via prostaglandin $\mathrm{E}(2)$-dependent reprogramming of host macrophages to increase their interleukin-10 production. Nat Med. 2009;15(1):42-49.

136. Tomchuck SL, et al. Toll-like receptors on human mesenchymal stem cells drive their migration and immunomodulating responses. Stem Cells. 2008;26(1):99-107.

137. Romieu-Mourez R, et al. Cytokine modulation of TLR expression and activation in mesenchymal stromal cells leads to a proinflammatory phenotype. J Immunol. 2009;182(12):7963-7973.

138. Chou DB, et al. Stromal-derived IL-6 alters the balance of myeloerythroid progenitors during Toxoplasma gondii infection. J Leukoc Biol. 2012;92(1):123-131.

139. Schurch CM, et al. Cytotoxic CD8+ T cells stimulate hematopoietic progenitors by promoting cytokine release from bone marrow mesenchymal stromal cells. Cell Stem Cell. 2014;14(4):460-472.

140. Shi C, et al. Bone marrow mesenchymal stem and progenitor cells induce monocyte emigration in response to circulating tolllike receptor ligands. Immunity. 2011;34(4):590-601.

141. Tse WT, et al. Suppression of allogeneic T-cell proliferation by human marrow stromal cells: implications in transplantation. Transplantation. 2003;75(3):389-397.

142. Di Nicola M, et al. Human bone marrow stromal cells suppress T-lymphocyte proliferation induced by cellular or nonspecific mitogenic stimuli. Blood. 2002;99(10):3838-3843.

143. Krampera M, et al. Bone marrow mesenchymal stem cells inhibit the response of naive and memory antigen-specific $\mathrm{T}$ cells to their cognate peptide. Blood. 2003;101(9):3722-3729.

144. Ferraro F, et al. Diabetes impairs hematopoietic stem cell mobilization by altering niche function. Sci Transl Med. 2011;3(104):104ra1

145. Yue R, et al. Leptin receptor promotes adipogenesis and reduces osteogenesis by regulating mesenchymal stromal cells in adult bone marrow. Cell Stem Cell. 2016;18(6):782-796.

146. Yamazaki S, et al. Nonmyelinating Schwann cells maintain hematopoietic stem cell hibernation in the bone marrow niche. Cell. 2011;147(5):1146-1158.

147. Katayama Y, et al. Signals from the sympathetic nervous system regulate hematopoietic stem cell egress from bone marrow. Cell. 2006;124(2):407-421. 
148. Mendez-Ferrer S, et al. Haematopoietic stem cell release is regulated by circadian oscillations. Nature. 2008;452(7186):442-447. 149. Scheiermann C, et al. Adrenergic nerves govern circadian leukocyte recruitment to tissues. Immunity. 2012;37(2):290-301.

150. Spiegel A, et al. Catecholaminergic neurotransmitters regulate migration and repopulation of immature human CD34+ cells through Wnt signaling. Nat Immunol. 2007;8(10):1123-1131.

151. Kalinkovich A, et al. Blood-forming stem cells are nervous: direct and indirect regulation of immature human CD34+ cells by the nervous system. Brain Behav Immun. 2009;23(8):1059-1065.

152. Hanoun M, et al. Neural regulation of hematopoiesis, inflammation, and cancer. Neuron. 2015;86(2):360-373.

153. Al-Sharea A, et al. Chronic sympathetic driven hypertension promotes atherosclerosis by enhancing hematopoiesis. Haematologica. 2019;104(3):456-467.

154. Taichman RS, Emerson SG. Human osteoblasts support hematopoiesis through the production of granulocyte colony-stimulating factor. J Exp Med. 1994;179(5):1677-1682.

155. Taichman RS, et al. Human osteoblasts support human hematopoietic progenitor cells in vitro bone marrow cultures. Blood. 1996;87(2):518-524.

156. Calvi LM, et al. Osteoblastic cells regulate the haematopoietic stem cell niche. Nature. 2003;425(6960):841-846.

157.Zhang J, et al. Identification of the haematopoietic stem cell niche and control of the niche size. Nature. 2003;425(6960):836-841.

158. Silberstein L, et al. Proximity-based differential single-cell analysis of the Niche to identify stem/progenitor cell regulators. Cell Stem Cell. 2016;19(4):530-543.

159. Lymperi S, et al. Strontium can increase some osteoblasts without increasing hematopoietic stem cells. Blood. 2008;111(3):1173-1181.

160. Marriott I, et al. Osteoblasts express the inflammatory cytokine interleukin-6 in a murine model of Staphylococcus aureus osteomyelitis and infected human bone tissue. Am J Pathol. 2004;164(4):1399-1406.

161. Littlewood AJ, et al. The modulation of the expression of IL-6 and its receptor in human osteoblasts in vitro. Endocrinology. 1991;129(3):1513-1520.

162. Marriott I, et al. Osteoblasts produce monocyte chemoattractant protein-1 in a murine model of Staphylococcus aureus osteomyelitis and infected human bone tissue. Bone. 2005;37(4):504-512.

163. Gasper NA, et al. Bacterium-induced CXCL10 secretion by osteoblasts can be mediated in part through toll-like receptor 4 . Infect Immun. 2002;70(8):4075-4082.

164. Marriott I, et al. Induction of Nod1 and Nod2 intracellular pattern recognition receptors in murine osteoblasts following bacterial challenge. Infect Immun. 2005;73(5):2967-2973.

165. Madrazo DR, et al. Signaling via Toll-like receptor 5 can initiate inflammatory mediator production by murine osteoblasts. Infect Immun. 2003;71(9):5418-5421.

166. Thomas T. The complex effects of leptin on bone metabolism through multiple pathways. Curr Opin Pharmacol. 2004;4(3):295-300.

167. Jurimae J, et al. Adiponectin is associated with bone mineral density in perimenopausal women. Horm Metab Res. 2005;37(5):297-302.

168. Wei S, et al. IL-1 mediates TNF-induced osteoclastogenesis. J Clin Invest. 2005;115(2):282-290.

169. Cenci S, et al. Estrogen deficiency induces bone loss by enhancing T-cell production of TNF-alpha. J Clin Invest. 2000;106(10):1229-1237.

170.Sims NA, et al. Glycoprotein 130 regulates bone turnover and bone size by distinct downstream signaling pathways. J Clin Invest. 2004;113(3):379-389.

171. Bruns I, et al. Megakaryocytes regulate hematopoietic stem cell quiescence through CXCL4 secretion. Nat Med. 2014;20(11):1315-1320.

172. Zhao M, et al. Megakaryocytes maintain homeostatic quiescence and promote post-injury regeneration of hematopoietic stem cells. Nat Med. 2014;20(11):1321-1326.

173. Nakamura-Ishizu A, et al. CLEC-2 in megakaryocytes is critical for maintenance of hematopoietic stem cells in the bone marrow. J Exp Med. 2015;212(12):2133-2146.

174. Jiang J, et al. Shear enhances thrombopoiesis and formation of microparticles that induce megakaryocytic differentiation of stem cells. Blood. 2014;124(13):2094-2103.

175. Beaulieu LM, Freedman JE. The role of inflammation in regulating platelet production and function: Toll-like receptors in platelets and megakaryocytes. Thromb Res. 2010;125(3):205-209.

176. Couldwell G, Machlus KR. Modulation of megakaryopoiesis and platelet production during inflammation. Thromb Res. 2019;179:114-120.

177. Haas S. Inflammation-induced emergency megakaryopoiesis driven by hematopoietic stem cell-like megakaryocyte progenitors. Cell Stem Cell. 2015;17(4):422-434.

178. Kimura H, et al. Interleukin-1 beta (IL-1 beta) induces thrombocytosis in mice: possible implication of IL-6. Blood. 1990;76(12):2493-2500.

179. Nakai S, et al. Interleukin-1 potentiates granulopoiesis and thrombopoiesis by producing hematopoietic factors in vivo. Life Sci. 1989;45(7):585-591.

180. Kaser A, et al. Interleukin-6 stimulates thrombopoiesis through thrombopoietin: role in inflammatory thrombocytosis. Blood. 2001;98(9):2720-2725.

181. Shiraki R, et al. Expression of Toll-like receptors on human platelets. Thromb Res. 2004;113(6):379-385.

182. D'Atri LP, et al. Expression and functionality of Toll-like receptor 3 in the megakaryocytic lineage. J Thromb Haemost. 2015;13(5):839-850.

183. Thon JN, et al. T granules in human platelets function in TLR9 organization and signaling. J Cell Biol. 2012;198(4):561-574

184. Beaulieu LM, et al. Regulatory effects of TLR2 on megakaryocytic cell function. Blood. 2011;117(22):5963-5974.

185. Cognasse F, et al. Toll-like receptor 4 ligand can differentially modulate the release of cytokines by human platelets. Br J Haematol. 2008;141(1):84-91.

186. Aslam R, et al. Platelet Toll-like receptor expression modulates lipopolysaccharide-induced thrombocytopenia and tumor necrosis factor-alpha production in vivo. Blood. 2006;107(2):637-641.

187. Freedman JE, et al. Relation of platelet and leukocyte inflammatory transcripts to body mass index in the Framingham heart 
study. Circulation. 2010;122(2):119-129.

188. Linge $\mathrm{P}$, et al. The non-haemostatic role of platelets in systemic lupus erythematosus. Nat Rev Rheumatol. 2018;14(4):195-213

189. Boilard E, et al. Platelets amplify inflammation in arthritis via collagen-dependent microparticle production. Science. 2010;327(5965):580-583.

190. Chow A, et al. Bone marrow CD169+ macrophages promote the retention of hematopoietic stem and progenitor cells in the mesenchymal stem cell niche. J Exp Med. 2011;208(2):261-271.

191. Winkler IG, et al. Bone marrow macrophages maintain hematopoietic stem cell (HSC) niches and their depletion mobilizes HSCs. Blood. 2010;116(23):4815-4828

192. Christopher MJ, et al. Expression of the G-CSF receptor in monocytic cells is sufficient to mediate hematopoietic progenitor mobilization by G-CSF in mice. J Exp Med. 2011;208(2):251-260.

193. Ludin A, et al. Monocytes-macrophages that express a-smooth muscle actin preserve hematopoietic cells in the bone marrow. Nat Immunol. 2012;13(11):1072-1082.

194. Kaur S, et al. Self-repopulating recipient bone marrow resident macrophages promote long-term hematopoietic stem cell engraftment. Blood. 2018;132(7):735-749.

195. Casanova-Acebes M, et al. Rhythmic modulation of the hematopoietic niche through neutrophil clearance. Cell. 2013;153(5):1025-1035.

196. Bowers E, et al. Granulocyte-derived TNF $\alpha$ promotes vascular and hematopoietic regeneration in the bone marrow. Nat Med. 2018;24(1):95-102.

197. Chen X, et al. Bone marrow myeloid cells regulate myeloid-biased hematopoietic stem cells via a histamine-dependent feedback loop. Cell Stem Cell. 2017;21(6):747-760.

198. Huizinga GP, et al. The collision of meta-inflammation and SARS-CoV-2 pandemic infection. Endocrinology. 2020;161(11):bqaa154.

199. Griffin C, et al Sexually dimorphic myeloid inflammatory metabolic responses to diet-induced obesity. Am J Physiol Regul Integr Comp Physiol. 2016;311(2):R211-R216

200. Crewe C, et al. An endothelial-to-adipocyte extracellular vesicle axis governed by metabolic state. Cell. 2018;175(3):695-708. 